\title{
Intraoperative Digestive System Injury
}

National Cancer Institute

\section{Source}

National Cancer Institute. Intraoperative Digestive System Injury. NCI Thesaurus. Code C78380.

Damage to any part of the digestive system during a surgical procedure. 